\title{
Correction to: Concurrent Hospice Care and Cancer-Directed Treatment for Advanced Lung Cancer and Receipt of Aggressive Care at the End of Life in the Veteran's Health Administration by CJ Presley et al. (DOI: 10.1089/jpm.2019.0485)
}

\begin{abstract}
fter publication of the article entitled, "Concurrent Hospice Care and Cancer-Directed Treatment for Advanced Lung A Cancer and Receipt of Aggressive Care at the End of Life in the Veteran's Health Administration" by CJ Presley et al, (J Palliat Med 2020;23:1038-1044; DOI: 10.1089/jpm.2019.0485) an inadvertent omission of the following funding information was identified, and has been added to version of record. The complete funding information is reproduced below, with the added grant information identified in bold:
\end{abstract}

\section{Funding Information}

This project was funded by grants from the Veterans Affairs Robert Wood Johnson Clinical Scholar Program, the Yale Lung SPORE Career Development Award (P50CA 196530), and the Claude D. Pepper Older Americans Independence Center at Yale School of Medicine (P30AG 021342) from the National Institute of Aging. Dr. C.J.P. is a Paul Calabresi Scholar supported by the OSU K12 Training Grant for Clinical Faculty Investigators (K12 CA133250).

The online version of this article has been corrected.

The authors regret this omission. 